\title{
Tailored Radiotherapy Treatment in Advanced Cervical Cancer. Early Experience of Robotics in Surgical Innovation
}

\author{
Mikel Gorostidi Pulgar*, Constance Goiri and Arantxa Lekuona \\ Department of Obstetrics and Gynecology, Hospital Universitario Donostia, Guipuzcoa, Spain
}

Received: November 14, 2013; Accepted: January 29, 2014; Published: February 13, 2014

*Corresponding author: Gorostidi M, Usandizaga 18-6²Ida, 20002 San Sebastian, Spain, Tel: 34-670302774; Fax: 34-943007808; E-mail: mgorostidi@ sego.es

\begin{abstract}
The cornerstone of treatment for advanced cervical cancer is chemotherapy and concomitant radiotherapy, extending the radiation field to the paraaortic area if affected nodes are present in the upper abdomen. The sensitivity of available imaging techniques, including Positron Emission Tomography (PET), is low, with an inadmissible false negative rate. Surgical staging is the best way to assess nodal status. Moreover there are some reports that suggest therapeutic benefit of paraaortic lymphadenectomy in cervical cancer.

The uncertainty regarding survival impact of pre-treatment para-aortic lymph node assessment results in different treatment alternatives, in many cases depending on locally available resources or surgeons' training in advanced laparoscopic procedures.

As far as better sensitivity is achieved, a paraaortic lymphadenectomy should be offered preferably via retroperitoneal route until renal vein by MIS (either laparoscopy or robotics) whenever available.
\end{abstract}

\section{Review}

The cornerstone of treatment for advanced cervical cancer is chemotherapy and concomitant radiotherapy, extending the radiation field to the paraaortic area if affected nodes are present in the upper abdomen, because surgery alone is not likely to be curative and these patients usually require adjuvant treatment, which is associated with a high incidence of morbidity when combined.

Nowadays there is insufficient evidence that pre-treatment surgical para-aortic lymph node assessment for locally advanced cervical cancer is beneficial. No randomized trials address this issue, and therefore definitive recommendations cannot be made [1]. The uncertainty regarding survival impact of pre-treatment para-aortic lymph node assessment results in different treatment alternatives, in many cases depending on locally available resources or surgeons' training in advanced laparoscopic procedures.

We discuss very briefly the main discussion topics and the contribution of robotics to advanced cervical cancer treatment.

\section{Nodal status and RT treatment}

The most important prognostic factors in cervical cancer are
FIGO stage and nodal involvement. The presence of positive paraaortic lymph nodes greatly conditions survival in these patients. In addition, the presence or absence of lymph node metastases guides treatment planning. Knowledge of aortic node status will tailor radiotherapy field.

Surgical staging is the best way to assess nodal status. The sensitivity of available imaging techniques is very low. Computer Tomography (CT) and Magnetic Resonance Imaging (MRI) have sensitivities of 50 and 56\%, respectively. Positron Emission Tomography (PET) is the best imaging technique to asses nodal spread and has $82 \%$ sensitivity and $95 \%$ specificity [2]. It is considered acceptable only in populations with a relatively high probability of lymph node metastasis [3]. The False Positive (FP) rate for PET/CT is 11\% [4], which results in considerable overtreatment. A treatment option in these patients is to forgo lymphadenectomy and extend radiotherapy fields in these patients. This is not adequate for radiological negative patients, as up to $27.2 \%$ would be undertreated.

The implications of undertreating these patients are life threatening because they will relapse as a result of incomplete treatment. The survival rate with histologically positive paraaortic lymph nodes treated with extended-field radiation therapy is as high as $50 \%$.

Furthermore there are some reports that suggest therapeutic benefit of paraaortic lymphadenectomy in cervical cancer $[5,6]$. And last year in a large meta analysis published in Lancet Oncology by Gouy et al. [7] report a $12 \%$ false negative for PET (and up to 22\% in those with uptake during PET of pelvic nodes). They suggest surgical staging in PET negative patients. They report similar survival between nodal metastasis of $5 \mathrm{~mm}$ or less (and treated with extension of RT) and PET negative ones, suggesting a positive therapeutic effect of surgery [8].

There is no available evidence of improved overall survival in patients with surgically tailored chemoradiation. A randomized trial is needed to resolve these questions. The multicenter international study LiLACS (Lymphadenectomy in Locally Advanced Cervical Cancer) directed by Frumovitz at MD Anderson [9], is a phase 3 clinical trial comparing surgical tailored chemoradiation versus PET/CT tailored chemoradiation, with 
overall survival as the primary endpoint. This study will need 8 years to recruit 600 patients, and intends to detect a $9 \%$ increase in overall survival. It will resolve these and other questions.

\section{Retroperitoneal route}

Retroperitoneal access affords a comfortable operating field with no bowel loops. It's especially indicated in morbid obesity, prior surgeries, surgical restaging and retroperitoneal recurrences [10].

It also benefits women with important intestinal distension, a small mesenteric root or who cannot tolerate the trendelenburg position [11].

Transperitoneal access is more difficult in our opinion, with an $8 \%$ conversion rate to open surgery, especially in obese patients [12].

Research on animal models has demonstrated reduced adhesion formation with retroperitoneal access [13]. It is also worth noting that any adhesions forming will not affect the bowel loops, unlike those caused by transperitoneal access. To our knowledge, there are no studies comparing the morbidity associated with these two access sites. It is accepted that there is a lower risk of intraoperative intestinal complications with this approach [14]. Furthermore, retroperitoneal access could hypothetically reduce intestinal complications in subsequent radiotherapy, though this has not been demonstrated.

A $9 \%(+/-7)$ rate of post-radiotherapy intestinal complications has been reported with a 5-year follow-up in the radiotherapy treatment of gynecological malignancies [15].

\section{Upper boundary}

There are several publications about location of nodal metastasis in paraaortic lymphadenectomy. The upper boundary of dissection, either the Inferior Mesenteric Artery (IMA) or the renal vein, is a subject of debate. Some surgeons perform lymphadenectomy only up to the IMA, because single metastasis above IMA are not common, particularly if pelvic PET is negative [16], while others perform it up to the renal vein, because there is a significant percentage of patients with isolated supramesenteric metastasis [17]. Lymphadenectomy extended up to the renal vein can double the number of nodes obtained [18]. We suggest lymphadenectomy up to the renal vein.

\section{Laparoscopy or robotics}

The advantages of robotics have been extensively described in many papers, but there are few publications that describe this procedure $[11,19,20]$ and no papers that focus on potential disadvantages.

The retroperitoneal paraaortic technique is performed within a very narrow surgical field. Collision of robotics arms can be a problem difficult to resolve. Location of trocars is also very important in classical laparoscopy but we have encountered a greater margin for error in our experience. Additionally, one more port is necessary for robotics. Robotic trocars are slightly larger, $8 \mathrm{mms}$ vs. $5 \mathrm{mms}$.
The trocar disposition we use is quite similar to the one we use in classical laparoscopy [21]. We have a wide range of robot triangulation, with no collisions and the assistant fits more comfortably (never at all with robotics).

There are no randomized trials comparing outcomes of robotic and classical laparoscopy paraaortic retroperitoneal lymphadenectomy. Blood loss, cost, number of nodes, surgical times etc. are unresolved questions that need for controlled trials. In January 2014 the largest series of robotic extraperitoneal paraaortic lymphadenectomy has been published by the group of Vall D'Hebron [22]. They obtained a higher number of nodes when robotics are used instead of classical laparoscopy comparing it with a retrospective series of their group by classical laparoscopy (17 vs $14, \mathrm{p}<0,05)$, with decreased blood loss $(20 \mathrm{ml}$ vs $90 \mathrm{ml}$, $p<0,05$ ) without differences on postoperative complications.

In our experience with robotics we haven't found great advantages over classical laparoscopy approach. We have also found it is slower, in part due to our docking learning curve. And we have encountered some problems: It's easy for the trocar to slip out in obese. It's also easy to lose sight of the instruments, they should always be visible in the screen. It's difficult to measure how much force is being used and it can be dangerous if too much traction is applied to vascular structures. Advanced sealing devices are not available for Da Vinci S, Intuitive Surgical (Sunnyvale, CA), so using a bipolar dissector and monopolar energy a better dissection and pedicle skeletonation are key factors.

Preserving ovarian arteries in premenopausal women is not always easy, particularly the left ovarian artery, but must be accomplished, and make an ovarian transposition in the same surgery. We suggest tunneling the ovarian pedicle under the peritoneum and passing and suturing them through a peritoneal window at each side as high as possible to preserve ovarian function and prevent internal hernias.

In the near future, more robotic systems will be available. They will be cheaper and they will improve, probably incorporating tactile feedback as well as other innovations. We believe it is a promising technology.

\section{Pelvic lymphadenectomy}

Some authors defend that chemotherapy and concomitant RT do not control a proportion of affected nodes, and reducing tumoral mass of by removing suspected nodes could improve survival [23]. But there is no evidence to suggest routine pelvic lymphadenectomy in advance cervical cancer.

Pelvic lymphadenectomy can be performed by classical transperitoneal laparoscopy [24]. Pelvic retroperitoneal access has also been described [23], but the surgical technique is more complicated and the theorical benefices of reducing the adhesion formation of this approach are more limited. There are no reports of robotic approach; we have partially procedures, which we completed transperitoneal due to difficulties.

Staging in advanced cervical cancer must be surgical, as this is the only accurate method of assessing lymph node status. 
Paraaortic lymphadenectomy up to the renal vein provides more complete information. There is no overall survival benefit, but the information provided will help tailor treatment and can have a positive effect by decreasing tumoral mass. Laparoscopy and robotics are both feasible and safe, with few complications. RT will not be delayed by the procedure and RT morbidity will not be affected.

Robotics is more expensive and there are still no randomized trials demonstrating its potential advantages.

\section{References}

1. Brockbank E, Kokka F, Bryant A, Pomel C, Reynolds K (2013) Pretreatment surgical para-aortic lymph node assessment in locally advanced cervical cancer. Cochrane Database Syst Rev 3: CD008217.

2. Choi HJ, Ju W, Myung SK, Kim Y (2010) Diagnostic performance of computer tomography, magnetic resonance imaging, and positron emission tomography or positron emission tomography/computer tomography for detection of metastatic lymph nodes in patients with cervical cancer: meta-analysis. Cancer Sci 101(6): 1471-1479.

3. Kang S, Kim SK, Chung DC, Seo SS, Kim JY, et al. (2010) Diagnostic value of 18F-FDG PET for evaluation of paraaortic nodal metastasis in patients with cervical carcinoma: A meta-analysis. J Nucl Med 51(3): 360-367.

4. Perez-Medina T, Pereira A, Mucientes J, García-Espantaleón M, Jiménez JS, et al. (2013) Prospective evaluation of 18-fluoro-2-deoxyd-glucose positron emission tomography for the discrimination of paraaortic nodal spread in patients with locally advanced cervical carcinoma. Int J Gynecol Cancer 23(1): 170-175.

5. Leblanc E, Narducci F, Frumovitz M, Lesoin A, Castelain B, et al. (2007) Therapeutic value of pretherapeutic extraperitoneal laparoscopic staging of locally advanced cervical carcinoma. Gynecol Oncol 105(2): 304-311.

6. Marnitz S, Köhler C, Roth C, Füller J, Hinkelbein W, et al. (2005) Is there a benefit of pretreatment laparoscopic transperitoneal surgical staging in patients with advanced cervical cancer? Gynecol Oncol 99(3): 536-544.

7. Gouy S, Morice P, Narducci F, Uzan C, Gilmore J, et al. (2012) Nodalstaging surgery for locally advanced cervical cancer in the era of PET. Lancet Oncol 13(5): e212-220.

8. Gouy S, Morice P, Narducci F, Uzan C, Martinez A, Rey A, et al. (2013) Prospective multicenter study evaluating the survival of patients with locally advanced cervical cancer undergoing laparoscopic para-aortic lymphadenectomy before chemoradiotherapy in the era of positron emission tomography imaging. J Clin Oncol 31(24): 3026-3033.

9. Frumovitz M, Querleu D, Gil-Moreno A, Morice P, Jhingran A, et al. (2013) Lymphadenectomy in locally advanced cervical cancer study (lilacs): Phase III clinical trial comparing surgical with radiologic staging in patients with stages IB2-IVA cervical cancer. J Minim Invasive Gynecol 21: 3-8.

10. Dargent D, Ansquer Y, Mathevet P (2000) Technical development and results of left extraperitoneal laparoscopic paraaortic lymphadenectomy for cervical cancer. Gynecol Oncol 77(1): 87-92.
11. Magrina JF, Kho R, Montero RP, Magtibay PM, Pawlina W (2009) Robotic extraperitoneal aortic lymphadenectomy: development of a technique. Gynecol Oncol 113(1): 32-35.

12.Abu-Rustum NR, Chi DS, Sonoda Y, DiClemente MJ, Bekker G, et al. (2003) Transperitoneal laparoscopic pelvic and para-aortic lymph node dissection using the argon-beam coagulator and monopolar instruments: an 8-year study and description of technique. Gynecol Oncol 89(3): 504-513.

13. Occelli B, Narducci F, Lanvin D, Querleu D, Coste E, et al. (2000) De novo adhesions with extraperitoneal endosurgical para-aortic lymphadenectomy versus transperitoneal laparoscopic para-aortic lymphadenectomy: A randomized experimental study. Am J Obstet Gynecol 183(3): 529-533.

14. Huang M, Slomovitz BM, Ramirez PT (2009) Transperitoneal versus extraperitoneal para-aortic lymphadenectomy in patients with cervical cancer. Rev Obstet Gynecol 2(2): 101-106.

15. Stanic S, Mayadev JS (2013) Tolerance of the small bowel to therapeutic irradiation: a focus on late toxicity in patients receiving para-aortic nodal irradiation for gynecologic malignancies. Int J Gynecol Cancer 2013 23(4): 592-597.

16. Vergote I, Amant F, Berteloot P, Van Gramberen M (2002) Laparoscopic lower para-aortic staging lymphadenectomy in stage IB2, II, and III cervical cancer. Int J Gynecol Cancer 12(1): 22-26.

17. Gil-Moreno A, Magrina JF, Pérez-Benavente A, Díaz-Feijoo B, SánchezIglesias JL, et al. (2012) Location of aortic node metastases in locally advanced cervical cancer. Gynecol Oncol 125(2): 312-314.

18. Köhler C, Tozzi R, Klemm P, Schneider A (2003) Laparoscopic paraaortic left-sided transperitoneal infrarenal lymphadenectomy in patients with gynecologic malignancies: technique and results. Gynecol Oncol 91(1): 139-148.

19. Narducci F, Lambaudie E, Houvenaeghel G, Collinet P, Leblanc E (2009) Early experience of robotic-assisted laparoscopy for extraperitoneal para-aortic lymphadenectomy up to the left renal vein. Gynecol Oncol 115: $172-174$

20. Vergote I, Pouseele B, Van Gorp T, Vanacker B, Leunen K, et al. (2008) Robotic retroperitoneal lower para-aortic lymphadenectomy in cervical carcinoma: First report on the technique used in 5 patients. Acta Obstet Gynecol Scand 87(7): 783-787.

21.Gorostidi M (2013) Robotic retroperitoneal paraaortic lymphadenectomy at Donostia university hospital. J Minim Invasive Gynecol doi: 10.1016/j.jmig.2013.10.004.

22. Díaz-Feijoo B, Gil-Ibáñez B, Pérez-Benavente A, Martínez-Gómez X, Colás E, et al. (2013) Comparison of robotic-assisted vs conventional laparoscopy for extraperitoneal paraaortic lymphadenectomy. Gynecol Oncol 132: 98-101.

23. Querleu D, Leblanc E, Castelain B (1991) Laparoscopic pelvic lymphadenectomy in the staging of early carcinoma of the cervix. Am J Obstet Gynecol 164(2): 579-581.

24. Querleu D, Ferron G, Rafii A, Bouissou E, Delannes M, et al. (2008) Pelvic lymph node dissection via a lateral extraperitoneal approach: description of a technique. Gynecol Oncol 109(1): 81-85. 